\title{
Utilization of Guidelines for Management of Severe Acute Malnutrition in Children Aged 6-59 Months in Busia County Referral Hospital
}

\author{
Samuel Mbugua ${ }^{1 *} \quad$ Angeline Kirui $^{2} \quad$ Margaret Muiva ${ }^{2}$ \\ 1.School of Nursing, Meru University of Science and Technology. P.O. Box 972-60200, Meru-Kenya. \\ 2.School of Nursing Sciences, University of Nairobi P.O. Box 19676-00202 Nairobi, Kenya
}

The funding is from the Linked-Strengthening Maternal, Newborn and Child Health (MNCH) Research Training in Kenya. The grant is linked to Partnership for Innovative Medical Education in Kenya (PRIME-K). The project was supported by Award Number 5R24TW008907 from the US National Institutes of Health. The content is solely the responsibility of the authors and does not necessarily represent the official views of the US National Institutes of Health.

Abstract

Background: Malnutrition is responsible for $35 \%$ of deaths among children under five years of age globally. The prevalence of severe acute malnutrition in Kenya is estimated to be 6\%. In Busia County Referral Hospital case fatality rate for children with severe acute malnutrition in 2014 was 26\%. World Health Organization (WHO) has developed guidelines for the management of severe acute malnutrition in children. The use of these guidelines in treatment of children with malnutrition reduces mortality related to malnutrition. Objective: The objective of the study was to assess the utilization of guidelines for management of severe acute malnutrition in children aged 6-59 months in Busia County Referral Hospital. Methods: This was cross-sectional, descriptive study. Purposive sampling was used to select the study sample. A checklist was used to verify documentation of guidelines for management of malnutrition in children from the in-patient files. An overall guidelines utilization scoring tool was developed and used to rate the overall utilization of the guidelines. Data on the supply of essential nutritional commodities was obtained through key informant interviews. Descriptive data was analysed using mean, mode and median. Findings were presented in form of frequency tables and bar charts. Results: A total of ninety-six (96) participants were recruited for the study. The mean age of the participants was 21.85 months. There was significantly higher proportion of children with MUAC less than $11.5 \mathrm{~cm}$ among 6 to 12 months 20(69.0\%) [OR=9.26; 95\%CI=2.82-30.39; $\mathrm{P}<0.001$ ] and 13 to 24 months $16(44.4 \%)$ [OR=3.33; $95 \% \mathrm{CI}=1.10-10.09 ; \mathrm{P}=0.033$ ] compared to those aged 25 to 59 months $6(19.4 \%)$. Mean weight had increased from $7.97 \mathrm{Kg}$ at admission to $8.45 \mathrm{Kg}$ at 7 days and this difference was significant $(\mathrm{p}<0.0001)$ after paired samples $t$ test was computed. The average utilization of the guidelines was $86.4 \%$. Conclusion and recommendations: Guidelines for management of severe acute malnutrition were adequately utilized in Busia County Referral Hospital. Periodic training of all health workers involved in the care of children with severe acute malnutrition was recommended to ensure optimum utilization of the guidelines for management of severe acute malnutrition.

Keywords: Utilization, Guidelines, Severe acute malnutrition, management, under five years.

DOI: $10.7176 / \mathrm{JHMN} / 86-03$

Publication date: February $28^{\text {th }} 2021$

\section{Background}

It is estimated that 162 million children under 5 years suffered from stunting while 51 million suffered from wasting worldwide in the year 2013 (IFPRI, 2014). Malnutrition is responsible for 35\% of deaths among children under five years of age (Black et al., 2008). Malnutrition causes 2.8 million deaths per year (WHO, 2014). The median case fatality rate for severe acute malnutrition ranges between 30-50\% (Deen et al., 2003).

The prevalence of severe acute malnutrition in Kenya is estimated to be $6 \%(\mathrm{MoH}, 2009)$. The prevalence of wasting and severe wasting in the Western Kenya is $2.3 \%$ and $1 \%$ respectively (KNBS, 2008-09). In Busia County Referral Hospital case fatality rate for children with severe acute malnutrition is above the target of $3 \%$ (MoH 2009)

Kenya adopted the WHO guidelines for the management of malnutrition in 2008. Evaluation of the application of these guidelines in the management of children with acute malnutrition has been done in several sites in Kenya. In Garissa Provincial General Hospital, for example, adherence to National guidelines for Integrated Management of Acute malnutrition was documented in five out of the eight steps (Warfa et al., 2013). Management of children with severe acute malnutrition was inadequate at Garissa Provincial General Hospital (Warfa et al. 2013). In Kenyatta National Hospital, the care of children with acute malnutrition often did not follow the WHO guidelines (Nzioki et al., 2008). The study was done to assess the utilization of national guidelines for management of severe acute malnutrition in children aged 6-59 months admitted in Busia County 
Referral Hospital.

\section{Methods}

\subsection{Study design}

The study was cross-sectional, descriptive study to establish utilization of guidelines for management of severe acute malnutrition in children aged 6-59 months admitted to Busia County Referral Hospital, Kenya. Busia County Referral Hospital is located in Busia County in the former Western Province. The hospital has a total bed capacity for inpatient at 185 , while the pediatric unit has 35 beds. Children admitted with severe malnutrition account for $3 \%$ of all paediatric admissions in the hospital.

\subsection{Study population}

Children aged 6-59 months were selected for the study. Children of this age are more prone to malnutrition due to early cessation of breastfeeding and wrong weaning practices. They are also at a high risk of childhood diseases such as diarrhoea, respiratory tract infections and parasitic infections which predispose them to undernutrition.

\subsection{Inclusion and exclusion criteria}

\subsubsection{Inclusion Criteria}

1. All children aged between 6-59 months with severe acute malnutrition admitted to Busia County Referral Hospital for treatment.

2. Consenting parents/guardians

\subsubsection{Exclusion criteria}

1. Children aged between 6-59 months who had chronic illness precipitating malnutrition at the time of the study were excluded from the study.

2. All participants whose guardians/ caretakers refused to consent for the study were excluded from the study.

\subsection{Sample size determination}

Sample size was calculated using Fisher's et al 2003, formula: $\mathrm{n}=\left[\mathrm{t}^{2} \times \mathrm{p}(1-\mathrm{p})\right] / \mathrm{m}^{2}$; where $\mathrm{n}=$ minimum sample size required, $\mathrm{t}=$ confidence level at $95 \%$ (standard value of 1.96$), \mathrm{p}=$ estimated prevalence of severe acute malnutrition in Kenya is $6 \%(\mathrm{MoH}, 2009))$ and $\mathrm{m}=$ margin of error at $5 \%(0.05)$

Sample size $=\left[1.96^{2} \times 0.06(1-0.06)\right] / 0.05^{2}$

$=86.67$

$=87$

\subsection{Sampling procedure}

Purposive sampling was used to recruit participants from the paediatric ward when they were admitted for management of severe acute malnutrition.

\subsection{Data collection}

Data was obtained at admission and on the seventh day post admission for each participant. A checklist was used to check documentation and implementation of guidelines for management of malnutrition in children by clinical staff and the guardians. A research assistant was recruited to help with data collection. Prior to the actual data collection, the research assistant was trained on how to collect data using the checklist.

Key informant interviews were conducted to obtain data on the supply of nutritional commodities in the study sites involved in the care of children within the hospital. The nutritionist in charge of the hospital and the one in charge of the inpatients as well as the nurse in charge of the paediatric ward were interviewed. The participants to the key informant interviews were given an information sheet explaining the purpose of the interview. They were then given a consent form which they signed voluntarily before participating in the interviews. An audio recorder was used to capture the proceedings of the interview.

Data concerning preparation of feeds by the clinical staff and nutritionists and measurement of feeds by the guardians was obtained by observation. An observation checklist was used to collect data at the feed preparation area. Parent/ guardians were observed as they collected for their children the feeds to see if they measured the right type and amount of feeds to give to the children.

\subsection{Data Analysis and Data presentation}

Data was first checked for accuracy and completeness when the checklists were filled. Audio tapes were transcribed to extract data obtained through key informant interviews. All quantitative data was entered into the computer using Microsoft excel. It was later analysed using statistical package for social sciences (SPSS) version 
20. The findings were presented using frequency tables, charts and graphs.

An overall guidelines utilization scoring tool was developed. The overall guidelines utilization score was computed using twenty (20) elements extracted from the guidelines for management of severe acute malnutrition. The score 1 was given to the option "yes" while score 0 on the scale represented the category "no".

\subsection{Ethical Considerations}

Clearance was sought from Kenyatta National Hospital /University of Nairobi Ethics and Research Committee. Permission to conduct research in Busia County was obtained from the Busia County Hospital administration. A written informed consent to participate in the study was obtained from the parents/guardians. The data obtained was kept in confidence with only the researcher having access to the filled checklists and key informant interview recordings. All checklists were coded. The research assistant was required to take a confidentiality pledge before data collection commenced.

\section{Results}

A total of ninety-six (96) children with severe acute malnutrition were included in the study. Key informant interview involved a nurse and two nutritionists. The results are presented in tables.

\subsection{Demographic data of the participants}

The mean age of the participants in this study was 21.85 months. The proportions of the participants in the age groups (6-12), (13-24) and (25-59) months were almost equal representing 29(30.2\%), 36(37.5\%), 31(32.3\%) respectively. Majority $62(64.4 \%)$ of the participants were males. The highest percentage $39(40.6 \%)$ of the participants were from Matayos Sub-county, which hosts Busia County Referral Hospital, followed by $30(31.3 \%)$ from Teso South Sub-county which borders Matayos Sub-county to the north as shown in Table 1.

Table 1. Demographic Data of the Participants

\begin{tabular}{|c|c|c|}
\hline Demographic data & Frequency, $(n=96)$ & Percent (\%) \\
\hline \multicolumn{2}{|c|}{ Mean age in months $( \pm \mathrm{SD}) \quad 21.85( \pm 13$} & \\
\hline \multicolumn{3}{|c|}{ Age in months } \\
\hline 6 to 12 & 29 & 30.2 \\
\hline 13 to 24 & 36 & 37.5 \\
\hline 25 to 59 & 31 & 32.3 \\
\hline \multicolumn{3}{|l|}{ Sex } \\
\hline Male & 62 & 64.6 \\
\hline Females & 34 & 35.4 \\
\hline \multicolumn{3}{|l|}{ Sub county } \\
\hline Matayos & 39 & 40.6 \\
\hline Samia & 4 & 4.2 \\
\hline Teso South & 30 & 31.3 \\
\hline Butere Mumias & 4 & 4.2 \\
\hline Siaya & 4 & 4.2 \\
\hline Uganda & 15 & 15.6 \\
\hline
\end{tabular}

\subsection{Anthropometric measurements among the participants}

The anthropometric measurements among the participants are presented in Table 2. The distribution of mean weight on admission, weight on day 7, weight gain, height and mid upper arm circumference (MUAC) among the participants who participated in the study was $7.97 \mathrm{~kg}, 8.44 \mathrm{~kg}, 0.47 \mathrm{~kg}, 76 \mathrm{~cm}$ and $11.6 \mathrm{~cm}$ respectively. More than half 54(56.3\%) had a MUAC score of greater or equal to $11.5 \mathrm{~cm}$ compared to $42(43.8 \%)$ who had less than $11.5 \mathrm{~cm}$. Out of the 96 participants, $57(59.4 \%$ ) had a weight for height $\mathrm{Z}$ score (WHZ) of less than $-3 \mathrm{SD}$ while $19(19.8 \%)$ had WHZ less than -1SD. Mean weight had increased from $7.97 \mathrm{Kg}$ at admission to $8.45 \mathrm{Kg}$ at 7 days and this differences was significant $(\mathrm{p}<0.0001)$ after paired samples $t$ test was computed.

Table 2. Anthropometric Measurements among the Participants

\begin{tabular}{|l|c|c|c|}
\hline \multicolumn{1}{|c|}{ Variables } & Mean(+SD) & Frequency, (n) & Percent, (\%) \\
\hline Mean weight on admission in kg & $7.97(+3.10)$ & - & - \\
\hline Mean weight on day 7 in kg & $8.44(+3.02)$ & - & - \\
\hline Mean weight gain in kg & $0.47(+0.59)$ & - & - \\
\hline Mean height in cm & $76(+13.92)$ & - & - \\
\hline Mean of MUAC in cm & $11.6(+1.53)$ & - & - \\
\hline MUAC
\end{tabular}




\begin{tabular}{|l|c|c|c|}
\hline \multicolumn{1}{|c|}{ Variables } & Mean(+SD) & Frequency, (n) & Percent, (\%) \\
\hline$<11.5$ & - & 42 & 43.8 \\
\hline$\geq 11.5$ & - & 54 & 56.3 \\
\hline WHZ score & - & 57 & 59.4 \\
\hline-3 SD & - & 20 & 20.8 \\
\hline-2 SD & - & 19 & 19.8 \\
\hline-1 Sd & \multicolumn{4}{|l}{} \\
\hline
\end{tabular}

3.3 Socio-demographic characteristics of guardians to the participant

The mean age of the guardians was 30.3 years. The findings also show that about one third of the guardians $36(37.5 \%)$ and $37(38.5 \%)$ were within the age groups of 17-24 years and 25-34 years respectively. The rest $23(24.0 \%)$ were 35 years and above. Most of the guardians were females $88(91.7 \%)$. Most of the guardians $83(86.5 \%)$ were Christians. Majority $63(65.6 \%)$ of the guardians had attained primary school education while $15(15.6 \%)$ had not attained any formal education. About two thirds $56(60.2 \%)$ of the guardians were housewives.

3.4 Association between socio-demographic characteristics of the participants and their MUAC

The relationship between socio-demographic characteristics and MUAC among participants with severe acute malnutrition is presented in Table 3 . There was significantly higher proportion of children with MUAC less than $11.5 \mathrm{~cm}$ among 6 to 12 months 20(69.0\%) [OR=9.26;95\%CI=2.82-30.39; $\mathrm{P}<0.001$ ] and 13 to 24 months $16(44.4 \%)[\mathrm{OR}=3.33 ; 95 \% \mathrm{CI}=1.10-10.09 ; \mathrm{P}=0.033]$ compared to those aged 25 to 59 months $6(19.4 \%)$. Parents/guardians who never attended school and those who attended primary education had more children with MUAC less than $11.5 \mathrm{~cm}$ than those who attended secondary school. This difference was not statistically significant. There was no significant association $(\mathrm{P}<0.05)$ observed between the other socio-demographic characteristics and level of MUAC.

Table 3. Association between Socio-Demographic Characteristics and MUAC of Participants with Severe Acute Malnutrition

\begin{tabular}{|c|c|c|c|c|c|c|}
\hline \multirow{2}{*}{$\begin{array}{c}\text { Socio-demographic } \\
\text { characteristics }\end{array}$} & \multicolumn{2}{|c|}{ MUAC } & \multirow{2}{*}{ OR } & \multicolumn{2}{|c|}{$95 \%$ CI } & \multirow{2}{*}{$\begin{array}{c}\text { Test } \\
\text { P value }\end{array}$} \\
\hline & $<11.5, \mathrm{n}(\%)$ & $>11.5, \mathrm{n}(\%)$ & & Lower & Upper & \\
\hline \multicolumn{7}{|l|}{ Child's age in months } \\
\hline 6 to 12 & $20(69.0 \%)$ & $9(31.0 \%)$ & 9.26 & 2.82 & 30.39 & $<0.001$ \\
\hline 13 to 24 & $16(44.4 \%)$ & $20(55.6 \%)$ & 3.33 & 1.10 & 10.09 & 0.033 \\
\hline 25 to 59 & $6(19.4 \%)$ & $25(80.6 \%)$ & 1.00 & & & \\
\hline \multicolumn{7}{|l|}{ Child's Sex } \\
\hline Male & $24(38.7 \%)$ & $38(61.3 \%)$ & 0.56 & 0.24 & 1.31 & 0.178 \\
\hline Females & $18(52.9 \%)$ & $16(47.1 \%)$ & 1.00 & & & \\
\hline \multicolumn{7}{|l|}{ Guardian's age in years } \\
\hline $17-24$ & $15(41.7 \%)$ & $21(58.3 \%)$ & 1.63 & 0.54 & 4.95 & 0.386 \\
\hline $25-34$ & $20(54.1 \%)$ & $17(45.9 \%)$ & 2.69 & 0.90 & 8.07 & 0.078 \\
\hline 35 and above & $7(30.4 \%)$ & $16(69.6 \%)$ & 1.00 & & & \\
\hline \multicolumn{7}{|l|}{ Guardian's sex } \\
\hline Male & $3(37.5 \%)$ & $5(62.5 \%)$ & 0.75 & 0.17 & 3.35 & 0.710 \\
\hline Female & $39(44.3 \%)$ & $49(55.7 \%)$ & 1.00 & & & \\
\hline \multicolumn{7}{|c|}{ Guardian's level of education } \\
\hline None & $8(53.3 \%)$ & $7(46.7 \%)$ & 4.00 & 0.89 & 18.01 & 0.071 \\
\hline Primary & $30(47.6 \%)$ & $33(52.4 \%)$ & 3.18 & 0.94 & 10.74 & 0.062 \\
\hline Secondary & $4(22.2 \%)$ & $14(77.8 \%)$ & 1.00 & & & \\
\hline \multicolumn{7}{|l|}{ Guardian's religion } \\
\hline Christian & $39(47.0 \%)$ & $44(53.0 \%)$ & 2.96 & 0.76 & 11.52 & 0.106 \\
\hline Muslim & $3(23.1 \%)$ & $10(76.9 \%)$ & 1.00 & & & \\
\hline \multicolumn{7}{|l|}{ Guardian's occupation } \\
\hline Housewife & $24(42.9 \%)$ & $32(57.1 \%)$ & 1.25 & 0.40 & 3.92 & 0.702 \\
\hline Jua Kali & $3(50.0 \%)$ & $3(50.0 \%)$ & 1.67 & 0.25 & 11.07 & 0.597 \\
\hline Housemaid & $5(71.4 \%)$ & $2(28.6 \%)$ & 4.17 & 0.61 & 28.62 & 0.147 \\
\hline Student & $2(25.0 \%)$ & $6(75.0 \%)$ & 0.56 & 0.08 & 3.69 & 0.543 \\
\hline
\end{tabular}

\subsection{Diagnostic and laboratory investigations and Comorbidities}


Blood slide for malaria parasites $(84.4 \%)$ and haemoglobin $(83.3 \%)$ level tests were the common laboratory investigations carried out for the participants. Others include urinalysis (35.4\%), provider-initiated testing and counseling (PITC) (30.2\%) for HIV, and liver function tests (LFTs) (13.5\%). Chest X ray was done for only $13.55 \%$ of the participants. Diarrhoea was the main comorbidity $19(27.5 \%)$ and among the multiple comorbidities diarrhoea and malaria were $8(11.6 \%)$. Other comorbidities include cough (14.5\%), pneumonia $(5.8 \%)$ and candidiasis $(5.8 \%)$.

\subsection{Prevention/treatment of hypoglycaemia}

Random blood sugar (RBS) level investigation on admission was not carried out for most $80(83.3 \%)$ of the participants. Among those who had RBS level measured, the majority $14(87.5 \%)$ had RBS level greater or equal to $3 \mathrm{mmol} /$ litre while only 2(12.5\%) were diagnosed with hypoglycaemia (RBS $<3 \mathrm{mmol} / \mathrm{litre}$ ). RBS level was repeated in 30 minutes for all who diagnosed with hypoglycaemia. About three quarters $82(77.1 \%)$ of the participants were started on feeding within 30 minutes and F75 was the main type of feed used for 80(97.6\%) children to initiate feeding immediately on admission. Only $7(8.5 \%)$ of the participants were given $10 \%$ dextrose as the initial feed.

\subsection{Prevention/ treatment of hypothermia}

Temperature on admission was taken for the majority $80(83.3 \%)$ of the participants while for $16(16.7 \%)$ it was not taken. The mean temperature reading for those whose temperature was taken on admission was 37.3 degree centigrade $\left({ }^{\circ} \mathrm{C}\right)$. Two participants $2(2.5)$ had hypothermia. All the guardians/caretakers were advised to keep child warm, to keep baby and bed dry. Similarly, all the participants were bathed while in the ward; all were kept warm by caretaker and all protected from draught. Most of the respondent $81(84.4 \%)$ used towels to dry the baby after taking a bath.

\subsection{Prevention /treatment of dehydration}

All participants were assessed for dehydration and majority $77(86.5 \%)$ were diagnosed with dehydration. Locally made resomal was the main fluid used for rehydration of most $68(88.3 \%)$ participants with dehydration and all of them were given resomal orally. Forty-two $(43.8 \%)$ of the participants were in hypovolaemic shock and all of them were treated through the intravenous route. Thirty-four $(80.9 \%)$ of the participants in hypovolaemic shock were treated using Ringers lactate with $5 \%$ dextrose while $8(19.1 \%)$ were treated using normal saline. For the participants diagnosed with dehydration, F75 was given in alternate hours with resomal after the first two hours of rehydration with resomal.

\subsection{Monitoring of respiration, pulse, urine output and weight gain during rehydration}

The study showed that $55 \%$ of the participants were monitored for respiration and pulse during rehydration. However, $94.4 \%$ and $65.0 \%$ of the participants were not monitored for weight gain and urine output respectively during rehydration.

\subsection{Correction of electrolyte balance and Checking for infection}

All of the participants did not receive magnesium supplementation. Only, 3(3.1\%) of the participants were supplemented with potassium. All the participants were treated for presumed bacterial infection. A combination of benzyl penicillin and gentamycin were the main $75(78.1 \%)$ drugs given to the participants. Ceftriaxone was only given to $2(2.1 \%)$ participants. Almost all $94(97.9 \%)$ of the participants were also monitored for increased desire to feed while undergoing treatment.

\subsection{Monitoring of micronutrients}

All the participants were clinically assessed for micronutrient deficiency and all of them were on F75/F100. However, large percentage $86(89.2 \%)$ of the participants were not supplemented with micronutrient. Among those who were treated for micronutrient deficiency, 2 participants $(20 \%)$ received folate together with zinc, 2 $(20 \%)$ received a single dose of vitamin A, $3(30 \%)$ got multivitamin syrup while the rest $3(30 \%)$ were give high dose vitamin A (day 1, 2 and 14) together with multivitamin syrup and zinc.

\subsection{Initial re-feeding}

All the participants were started on re-feeding at admission and majority 72(75.0\%) were fed every 3 hours. F75 was the most common type of feed $86(89.6 \%)$ used for initial re-feeding. F100 was used for $7(7.3 \%)$ participant while PlumpyNut was used for 3(3.1\%) participants in the initial re-feeding. All of the participants were monitored for daily body weight and vomiting during initial re-feeding. However, more than half 55(57.3\%) were not monitored for frequency and consistency stool during initial re-feeding. 


\subsection{Monitor Catch-up growth}

Among those who given F75 for initial re-feeding, F100 was used for replacement in the transition phase. The volume of the replacement feed was increased after one day for most $79(91.9 \%)$ of the participants while for the rest $7(8.1 \%)$ it was increased after 2 days. Most $82(95.4 \%)$ of the participants had their replacement feed increased by $10 \mathrm{ml}$ daily. Pulse and respiration were monitored for $16(18.6 \%)$ of the participants during catch-up growth. Daily weighing in the morning before feeding was done for all participants. However, none of the participants had the weight gain calculated and recorded.

\subsection{Assure sensory stimulation and Prepare for follow up}

Data was sought as to whether the hospital exposes children with severe acute malnutrition to structured play, duration of structured play if it is provided and whether the children are provided with suitable toys for play. All these were not available in the hospital. Preparation for follow up was done to all the children admitted with severe acute malnutrition by discharging them through the nutrition clinic where the parents/guardians were informed of the follow up schedule. The participants were also issued with nutritional supplements on discharge from the inpatient care.

\subsection{Level of utilization of guidelines}

Twenty (20) variables regarding the utilization of guidelines for severe acute malnutrition were used to determine the level of utilization (Appendix I). The overall scores for utilization of guidelines ranged from 16 to 20. Percentage score was computed for each participant and the average utilization of the guidelines was $86.4 \%$. Moreover, there was high $(62.5 \%)$ and very high $(37.5 \%)$ utilization of the guidelines among the children with severe acute malnutrition.

\section{Discussion}

Guidelines for management of severe acute malnutrition were adequately utilized in Busia County Hospital. The major areas where deficiencies in the utilization were found include treatment/prevention of hypoglycaemia, prevention/treatment of hypothermia, prevention/treatment of dehydration and monitoring of catch up growth.

Random blood sugar level investigation on admission was not carried out for most of the participants $(83.3 \%)$ in this study. This finding compares with a study carried out at a national referral hospital in Kenya where random blood sugar was measured for $29.9 \%$ of the participants (Nzioki et. al. 2008). As indicated by KIIs, the hospital may at some point in time have lacked a working glucometer and glucostrips for measurement of random blood sugar explaining why random blood sugar was done for only a few patients. The participants found to have hypoglycaemia (12.5\%) were appropriately treated with $10 \%$ dextrose and random blood sugar repeated after thirty (30) minutes of treatment. Initiation of feeding was done immediately on admission for most participants $(77.1 \%)$ as required by the Ministry of Health, Kenya $(\mathrm{MoH}, 2013)$. This is in contrast to the finding at Kenyatta National Hospital where feeding was delayed for a median waiting time of 14.7 hours (Nzioki et al., 2008) and Garissa where feeds were initiated after an average of 2.6 hours (Warfa et al., 2013). Availability of feeds and efforts by the nutritionists in the hospital may have contributed to the early initiation of feeding. Hypoglycaemia as well as hypothermia, dehydration and severe infections occur soon after admission of severely malnourished children $(\mathrm{MoH}, 2013)$. These complications should be addressed urgently to prevent death $(\mathrm{MoH}, 2009)$.

In this study temperature on admission was taken for the majority of the participants $(83.3 \%)$. This is consistent with the guidelines which require all children with severe acute malnutrition to be monitored for hypothermia. In children suffering from severe acute malnutrition, both heat generation and heat loss are impaired; the child becomes hypothermic in a cold environment and hyperthermic in a hot environment. These findings are different from results of a study at Kenyatta National Hospital where monitoring of temperature was rarely done at admission or during hospitalization (Nzioki et al., 2008). The two (2) participants detected with hypothermia were appropriately managed using a heater available in the ward. However, subsequent monitoring for hypothermia was not done for them and other participants admitted to the ward as was the case in Kenyatta National Hospital (Nzioki et. al, 2008). This can be attributed to knowledge and skill gap since not all health workers involved in care of children with malnutrition are trained on management of severe acute malnutrition (KII 1\&2). All the guardians/caretakers in this study were appropriately advised to keep the participants warm as outlined in the guidelines (MoH 2009) by heavily dressing the children. This doesn't compare at all with Garissa where only $11.1 \%$ of OPD patients and $10 \%$ of in-patients had "keep warm" prescribed in their treatment (Warfa et. al, 2013). This finding can be due to the availability of clinical staff and nutritionists in Busia county Referral Hospital who are trained in the management of severe acute malnutrition.

This study shows that all participants were assessed for dehydration and majority $77(86.5 \%)$ were diagnosed with dehydration. The percentage of participants diagnosed with dehydration is close to the findings in Garissa where $65 \%$ had dehydration at the time of admission (Warfa et. al, 2013). A lower percentage of 
participants $(27.5 \%)$ in this study had diarrhoea when compared to findings from Kenyatta National Hospital where 64 out of 101 children had diarrhoea (Nzioki et. al, 2008). Eight participants (19.1\%) in hypovolaemic shock were incorrectly treated using normal saline in Busia County Hospital which reflects a lower rate of inappropriate treatment for hypovolaemic shock when compared to the practice in Kenyatta National Hospital where most children in hypovolaemic shock were treated with normal saline contrary to the guidelines (Nzioki et. al, 2008). This disparity may be due to increased knowledge on management of severe acute malnutrition in Busia County Hospital over time since the two studies were done some (7) years apart.

Correct treatment for dehydration using locally made "resomal" was done for $68(88.3 \%)$ of the participants with dehydration. This correlates with the availability of locally made "resomal" in Busia County Referral Hospital. However, not all participants were monitored during rehydration. Slightly more than half of the participants $(55 \%)$ treated for dehydration had pulse and respiration monitored during rehydration therapy as per the guidelines. This compares fairly to finding in Garissa where correct monitoring of rehydration was done for $43.3 \%$ of patients in the ward (Warfa et al., 2013) and contrasts findings in Kenyatta National Hospital where monitoring for signs of over hydration was rarely done (Nzioki et. al, 2008).

Children having severe acute malnutrition often have a serious electrolyte imbalance which may manifest at any time during treatment. Treatment of electrolyte imbalance is done using F75 which contains macro- and micronutrients in quantities that are enough to correct the imbalance. In this study only $5(5.2 \%)$ participants with severe acute malnutrition were investigated for electrolytes while $3(3.1 \%)$ of the participants were supplemented with potassium. In a study done in Pakistan, $93(63.3 \%)$ of children were investigated for electrolyte imbalance which represents a big difference compared to this study (Younas et al. 2012). All participants in our study were appropriately treated with F75 without supplementation of magnesium. This shows an improved utilization of guidelines when compared to Kenyatta National Hospital where approximately $56(55 \%)$ of participants were given commercially prepared F75 (Nzioki et. al, 2008).

All severe acute malnourished children are treated with antibiotic upon admission, regardless of whether they have clinical signs and symptoms of systemic infection or not. The antibiotic administered for such routine treatment must be active against small bowel bacterial overgrowth $(\mathrm{MoH} 2009)$. In this study, all the participants were given drugs for presumed bacterial infection as recommended in the guidelines. Younas et al. (2012) found the same rate of treatment for presumed bacterial infection whereby all children having severe acute malnutrition received broad spectrum antibiotics at admission. However, Nzioki et al., (2008) in their study reported a slightly lower percentage of children getting routine treatment for presumed bacterial infection since only $90 \%$ of children received broad spectrum antibiotics as per WHO recommendations. Since the prescription of antibiotics is done by the doctor who examines the child, it is likely that this step would not to be missed and hence all the participants were given treatment for presumed bacterial infection.

F75, F100, RUTF and locally-developed milk with combined mineral vitamin (CMV) mix provide the adequate amount of Vitamin A to manage mild Vitamin A deficiency and to replace low liver stores of Vitamin A during treatment. High dose Vitamin $\mathrm{A}$ is given if a patient has signs of severe deficiency. In this study 3 (3.1\%) were correctly treated with high dose vitamin A while 2 patients were given single doses of vitamin A even without clinical signs of severe vitamin A deficiency at the time of admission. Similarly, 5 (5.2\%) patients were given zinc against the guidelines, since the guidelines advise that zinc should not be given to children with malnutrition if they are receiving F75, F100or RUTF. Warfa et al. (2013) found out that a higher number of patients 78/96 (81.3\%) received correct dose of Vitamin A in Garissa. Likewise, 56 (55\%) children received high dose vitamin A on day one in Kenyatta National Hospital (Nzioki et. al, 2008). These disparities in the use of vitamin A can be due to the availability of guidelines for use in Busia County Referral Hospital which state clearly when these micronutrients should be given to children with severe acute malnutrition. The guidelines were issued by the Ministry of Health (MoH 2009) in the year 2009 and patients at Kenyatta National Hospital may not have benefitted from them when Nzioki et al., (2008) carried out their research.

In this study, F75 was the most common type of feed $86(89.6 \%)$ used for initial re-feeding. This is consistent with the guidelines of the Ministry of Health, Kenya (MoH 2013) which advises use of F75 to initiate re-feeding. These findings are supported by the fact that F75 was available in the hospital during the period of the study (KII 1- Nutritionist; KII -Nursing). Also, most of the prescription for F75 was made by the nutritionists in the ward which may imply that since the nutritional care was their responsibility, all the children could benefit from their service. Nzioki et al., (2008) had different findings in Kenyatta National Hospital where only 55\% of children were fed with F75 in the initial phase though premixed formula was available. Warfa et al., (2008) found that on average, children received their first feed 2.6 hours after admission (Warfa et al 2013). Our study showed more favourable results when compared to that of Warfa et al., (2013) since most 82 (77.1\%) of the participants received their feed within the first 30 minutes of admission.

In the rehabilitation phase a vigorous approach to feeding is required to achieve very high intakes and rapid weight gain of $>10 \mathrm{~g}$ gain $/ \mathrm{kg} / \mathrm{d}$. Milk-based F-100 is recommended in this phase. Among all those participants $(86,87.5 \%)$ who were given F75 for initial re-feeding, F100 was used as the replacement feed according to the 
guidelines, in volumes equal to that of F75 for 48 hours at the end of acute phase. However, the volume of F100 after transition period was routinely increased after one day for most participants. This is inconsistent with the guidelines which recommend that volume of F100 be increased by $10 \mathrm{ml}$ per feed until some feed remains uneaten (MoH, 2009).

\section{Conclusion and recommendations \\ 5.1 Conclusion}

The severity of malnutrition was associated with the age of the participants. There was significantly higher proportion of children with MUAC less than $11.5 \mathrm{~cm}$ among 6 to 12 months $20(69.0 \%)$ [OR=9.26; $95 \% \mathrm{CI}=2.82$ $30.39 ; \mathrm{P}<0.001]$ and 13 to 24 months $16(44.4 \%)$ [OR=3.33; 95\% CI=1.10-10.09; $\mathrm{P}=0.033$ ] compared to those aged 25 to 59 months $6(19.4 \%)$ as shown in Table 4.5 .

Guidelines for management of severe acute malnutrition were adequately utilized in Busia County Referral Hospital. The overall scores for utilization of guidelines ranged from 16 to 20 (Figure 4.5). The average utilization of the guidelines was $86.4 \%$.

Commodities needed for management of children with malnutrition, particularly F75 and F100 were available at Busia County Referral Hospital. Key informant interviews showed that commodities needed for management of severe acute malnutrition in the hospital were in stock most of the time. Also, the ingredients needed for making resomal in the hospital were consistently availed to the nutritionist by the hospital.

Some health workers involved in the care of children with severe acute malnutrition in Busia County Referral Hospital were not adequately trained in management of severe acute malnutrition. Integrated case management of acute malnutrition training was conducted for a small proportion of health workers in 2013 . Other health workers have not undergone this training.

\subsection{Recommendations}

Continuous medical education for all health workers on Case management of severe acute malnutrition.

The hospital management should maintain reliable supply of commodities needed for management of severe acute malnutrition.

A study should be done to evaluate the effectiveness of utilization of guidelines for management of severe acute malnutrition with a focus on staff to patient ratios.

\section{Competing interests}

The authors declare no competing interests

\section{References}

Black R E, Lindsay H. A., Zulfiqar A. B., Laura E. C., Mercedes de O., Majid E., Colin M., Juan R.(2008). Maternal and child undernutrition: global and regional exposures and health consequences. Lancet, 371:243-260.

Deen J. L., Blössner, M, de Onis, M. (2005) Malnutrition: quantifying the health impact at national and local levels. Geneva, World Health Organization. (W.H.O. Environmental Burden of Disease Series, No. 12).

International Food Policy Research Institute (2014). Global Nutrition Report 2014: Actions and Accountability to Accelerate the World's Progress on Nutrition. Washington, DC.

Kenya National Bureau of Statistics (KNBS) and ICF Macro. 2010. Kenya Demographic and Health Survey 2008-09. Calverton, Maryland: KNBS and ICF Macro.

Manary M. J., and Sandige H. L., (2008). Management of acute moderate and severe childhood malnutrition. BMJ.337, a2180.

Ministry of Health (2009). National guidelines for Integrated Management of Acute Malnutrition Version 1.Nairobi, Ministry of Health.

Ministry of Health (2013). Basic Paediatric Protocols for ages up to 5 years, November 2013 edition. Nairobi, Ministry of Health.

Nzioki C., Grace I., Rachel M., and Mike E., (2008). Audit Of Care For Children Aged 6 To 59 Months Admitted With Severe Malnutrition At Kenyatta National Hospital, Kenya. Unpublished Masters Dissertation, Nairobi, University of Nairobi

Warfa O., Njai D., Ahmed L., Admani B., Were F., Wamalwa D., Osano B., Mburugu P., and Mohamed M. (2013). Evaluating the level of adherence to Ministry of Health guidelines in the management of Severe Acute Malnutrition at Garissa Provincial General Hospital, Garissa, Kenya. The Pan African Medical Journal. 2014; 17:214

WHO (1999). Management of severe malnutrition: a manual for physicians and other senior health workers. Geneva, WHO.

WHO (2013) Pocket Book of Hospital Care of Children: Guidelines for the Management of Common Childhood 
Illnesses (2nd edition) Geneva, WHO.

WHO/FAO (2014). Countries Vow to Combat Malnutrition through Firm Policies and Actions. Available at: https://www.who.int/mediacentre/news/releases/2014/icn2-nutrition/en/ Accessed on $18^{\text {th }}$ April 2015.

Younas M., Khan M. A., Khan,J., Shah, F. and Munir, A. (2012). Systematic Analysis of In-Patients' Care of Severely Malnourished Children at Hayatabad Medical Complex Peshawar: A Tool to Improve Quality of Care. Gomal Journal of Medical Sciences January-June 2012, Vol. 10, No. 1

Appendix I: Overall Guidelines Utilization Scoring Tool

The overall guidelines utilization score was computed using twenty (20) elements extracted from the guidelines for management of severe acute malnutrition. The score 1 was given to the option "yes" while score 0 on the scale represented the category "no". The following elements of guideline were assessed;

Prevention/treatment of hypoglycaemia

1. Measuring of RBS on admission $(\mathrm{Yes}=1, \mathrm{No}=0)$

2. Immediate initiation of feeding within 30 minutes $(\mathrm{Yes}=1, \mathrm{No}=0)$

Prevention/treatment of hypothermia

3. Measuring of temperature on admission $(\mathrm{Yes}=1, \mathrm{No}=0)$

4. Advice given to caretaker to keep child warm $(\mathrm{Yes}=1, \mathrm{No}=0)$

5. Keeping baby warm by caretaker $(\mathrm{Yes}=1, \mathrm{No}=0)$

6. Protecting child from draught ( $\mathrm{Yes}=1, \mathrm{No}=0$ )

7. Advice given to keep baby and bed dry $(\mathrm{Yes}=1, \mathrm{No}=0)$

8. Whether child is bathed while in the ward ( $\mathrm{Yes}=1, \mathrm{No}=0)$

9. Drying the child after bathing ( $\mathrm{Yes}=1, \mathrm{No}=0$ )

Prevention or treatment of dehydration

Checking for infection

10. Assessment for dehydration/shock $(\mathrm{Yes}=1, \mathrm{No}=0)$

11. Treatment for presumed bacterial infection $(\mathrm{Yes}=1, \mathrm{No}=0)$

12. Monitoring of appetite $(\mathrm{Yes}=1, \mathrm{No}=0)$

Monitoring of micronutrients

13. Clinical assessment for micronutrient deficiency $(\mathrm{Yes}=1, \mathrm{No}=0)$

14. Whether child was given F75/F100 ( $\mathrm{Yes}=1, \mathrm{No}=0$ )

Initial re-feeding

15. Whether child was started on re-feeding ( $\mathrm{Yes}=1, \mathrm{No}=0)$

16. Monitoring of vomiting during initial re-feeding (Yes $=1, \mathrm{No}=0$ )

17. Monitoring of frequency and consistency of stool during initial re-feeding $\left(\mathrm{Yes}=1, \mathrm{No}_{\mathrm{O}}=0\right)$

18. Monitoring of daily body weight during initial re-feeding $(\mathrm{Yes}=1, \mathrm{No}=0)$

Monitor Catch-up growth

19. Daily weighing in the morning before feeding $(\mathrm{Yes}=1, \mathrm{No}=0)$

20. Calculating and recording of weight gain $(\mathrm{Yes}=1, \mathrm{No}=0)$

The overall score was generated by aggregating the scores. The maximum attainable score was 20 . A percentage score was generated and classified as Low $(<50 \%)$, moderately high $(50-69 \%)$, High $(70-85 \%)$, and very high $(>85 \%)$. 